\title{
A influência da Gestão do Tempo sobre a Qualidade de Vida no Trabalho
}

\section{The influence of Time Management on Quality of Life at Work}

\author{
CAROLINA BARBOSA MONTENEGRO \\ Universidade Potiguar (UNP) \\ HERTA CAMILA FERNANDES DIÓGENES NUNES MAIA \\ Universidade Potiguar (UNP) \\ IZA LEILLA CARLOS DA SILVA \\ Universidade Potiguar (UNP) \\ JULIO CESAR FERRO DE GUIMARÃES \\ Universidade Federal de Pernambuco (UFPE) \\ ELIANA ANDRÉA SEVERO \\ Centro Universitário UniFBV/Wyden.
}

\section{RESUMO}

A gestão do tempo vem ganhando importância e sendo percebida como fator que interfere na qualidade de vida no trabalho. O estudo tem como objetivo analisar a influência da gestão do tempo sobre a qualidade de vida no trabalho em diferentes ramos de atividades no Nordeste brasileiro. Trata-se de uma pesquisa quantitativa (survey) aplicada a 454 gestores. O estudo foi concentrado na área de gestão do tempo utilizando três construtos: circunstância/desperdício, importância e urgência. Foram aplicados testes estatísticos de confiabilidade e normalidade dos dados para averiguar as correlações entre as variáveis. $\mathrm{Na}$ análise descritiva foram utilizadas Análise Fatorial Confirmatória, correlação de Pearson e a Regressão Linear Múltipla. A pesquisa trouxe como resultados que a qualidade de vida no trabalho é influenciada diretamente pela gestão do tempo por meio dos constructos estudados. Outro achado da pesquisa, trata da constatação de que o tempo é melhor aproveitado com as coisas importantes quando não se está dedicado às circunstâncias/desperdícios e urgências. Palavras-chave: Qualidade de Vida no Trabalho. Gestão do Tempo. Gestão de Pessoas. 


\section{Abstract}

Time management has been gaining importance and being perceived as a factor that interferes with quality of life at work. The study aims to analyse the influence of time management on quality of life at work in different branches of activity in the Brazilian Northeast. This is a quantitative survey applied to 454 managers. The study focused on the Time Management area using three constructs: circumstance/waste, importance and urgency. Statistical tests of reliability and normality of the data were applied to verify the correlations between the variables. In the descriptive analysis we used Confirmatory Factor Analysis, Pearson correlation and Multiple Linear Regression. The research brought as results that the quality of life at work is directly influenced by time management through the studied constructs. Another research finding is that time is better spent on important things when not dedicated to circumstances/waste and urgencies. Keywords: Quality of Life at Work. Time Management. Human Resources.

\section{INTRODUÇÃo}

Além das evidentes e necessárias transformações no ambiente organizacional para melhor garantir a eficiência produtiva, ao longo dos tempos a ênfase passou a ser nas pessoas e em como capacitá-las para o melhor resultado.

As organizações vêm se adaptando às tendências e necessidades de um mundo do trabalho que evoluiu em termos de ambiente, formas de produção e mercado consumidor interno e externo (LIMONGI-FRA NÇA, 2004). Para Guest (1979), esse processo organizacional de maximizar o potencial criativo das pessoas, envolvendo-as nas decisões que afetam sua vida laboral, é o que ele chama de qualidade de vida no trabalho (QVT). Yeo e Li (2012) também correlacionam o engajamento no trabalho e a condução de atividades específicas da função com a percepção que os trabalhadores possuem sobre a qualidade de vida no trabalho (QVT).

Considerando que o trabalho corresponde a apenas uma esfera da vida do sujeito, Wang, Wei-Ching, Kao, Huan e Wu (2010) ressaltam que as pessoas teriam tempo para se dedicar a outros aspectos de suas vidas. Dessa forma, a dimensão temporal vem ga- 
nhando espaço no ambiente organizacional devido à ampliação da concorrência global e o aumento das exigências de disponibilidade imediata de produtos e serviços (ORLIKWSKY; YATES, 2002). Além disso, o aumento do ritmo de vida voltado para fazer as coisas mais rápido, contraindo o tempo gasto e as ações necessárias para tal (GRARHMMER, 2002), geram pressão entre os empregados pela entrega de mais trabalho em menos tempo (JACKSON; MARTIN, 1996; PALMER; SCHOORMAN, 1999; TEUCHMANN; TOTTERDELL; PARKER, 1999; MAJOR, KLEIN; EHRHART, 2002).

Aliada à velocidade com que os sistemas podem coletar e processar informações em tempo real, a globalização possibilitou a participação das organizações em várias atividades, em diversos lugares, ao mesmo tempo (MYSZEWSKI, 2015). Assim, a gestão do tempo (GT) tem sua importância reconhecida na literatura organizacional (CLAESSENS; VAN EERDE; RUTTE; ROE, 2007; AEON; AEON; AGUINIS; DAVIS; DETERT; GLYNN; JACKSON; KOCHAN; KOSSEK; LEANA; MORRINSON; PEARCE; PFEFFER; ROUSSEAU; SUTCLIFFE, 2017). Wang et al. (2010), sob a justificativa de estudos anteriores, reforça a indicação da GT como essencial na diminuição do estresse laboral, podendo aumentar a satisfação no trabalho e melhorar a qualidade de vida no trabalho.

Discussões a respeito da necessidade de melhorar o tempo são incorporadas em modelos teóricos e intervenções práticas (GEORGE; JONES, 2000; ANCONA; OKHUYSEN; PERLOW, 2001; GRANT; CHRISTIANSON; PRICE, 2007; K ̈̈SER; FISCHBACHER; KÖNIG, 2013; HÄFNER; STOCK; OBERST, 2015; A GUINIS et al., 2016) e outros voltados para como as pessoas gerenciam seu tempo nas organizações (BLUEDORN; DENHARDT, 1988; MACAN, 1994; ANCONA et al., 2001; MYSZEWSKI, 2015).

O problema de como gerir o tempo é algo discutido e vários autores propõem métodos para lidar com questões relacionadas ao tempo no trabalho (MCCAY, 1959; DRUKER, 1967; MACKENZIE, 1972; LAKEIN, 1973; BARBOSA, 2011; COVEY, 2014). No geral, como afirma Koch e Kleinmann (2002), falar de GT é entender o que as pessoas fazem com o seu tempo em prol da obtenção de algo.

A necessidade de conciliar o tempo entre as atividades laborais e todas as outras funções sociais do indivíduo configura-se como 
justificativa desse estudo, que objetiva analisar a influência da gestão do tempo sobre a qualidade de vida no trabalho em diferentes ramos de atividades no Nordeste do Brasil.

\section{REFERENCIAL TEÓRICO}

\subsection{Qualidade de vida no trabalho}

Embora o trabalho seja positivo e benéfico para a vida do homem, sendo sua fonte de sustento e de socialização, muito se tem estudado sobre os seus efeitos na qualidade de vida, uma vez que corresponde a uma atividade que demanda grande dedicação de tempo, sob pressões por prazos e resultados (MYSZEWSKI, 2015; DE PAOLA; GIOIA, 2016).

De um lado, as organizações buscam identificar, planejar e implantar as melhores formas de trabalho que possibilitem aumentar a satisfação e bem-estar dos funcionários para que esses resultados continuem atendendo aos objetivos financeiros e de produção (D AL FORNO; FINGER, 2015). Esse papel do funcionário como essencial para contribuir e agregar valor ao sucesso organizacional, motivo pelo qual deveria ser tratado com respeito e dignidade, constitui, segundo Yeo e Li (2012), a base conceitual da QVT.

Segundo Nadler e Lawler III (1983), após os primeiros estudos de um grupo americano de pesquisadores, ao final da década de 1960, o interesse pela QVT foi retomado quase uma década depois com o aumento da concorrência internacional, que despontava como fenômeno em gestão e efetividade.

O conceito de QVT foi se modificando com o tempo, revelando uma indefinição do termo na literatura, o que pode levar ao desentendimento e desinteresse por parte da gestão em levar adiante projetos sobre QVT (NADLER; LAWLER III, 1983; DAL FORNO; FINGER, 2015).

Walton (1973) descreve um modelo de análise dos elementos que compõem a QVT a partir de oito categorias conceituais: i) Compensação adequada e justa; ii) Condições de segurança e de saúde no trabalho; iii) Oportunidade imediata para utilizar e desenvolver as capacidades humanas; iv) Oportunidade futura para contínuos 
crescimento e segurança; v) Integração social na organização de trabalho; vi) Constitucionalismo na organização de trabalho (normas estabelecendo direitos e deveres, evitando arbitrariedades); vii) Trabalho e espaço total de vida (balanço, equilíbrio entre as esferas trabalho/família); viii) A relevância social da vida no trabalho.

As inter-relações entre as oito categorias podem estar positivas ou negativamente associadas a depender do quanto cada categoria necessitará declinar de sua qualidade em detrimento de outra. A dedicação requerida por oportunidades futuras, por exemplo, pode impactar no equilíbrio vida familiar/trabalho (WALTON, 1973). A conexão de elementos envolvidos na QVT também é evidenciada por Yeo e Li (2012), quando citam que os aspectos do trabalho podem impactar na vida pessoal, prejudicando o equilíbrio entre ambos.

As atividades voltadas para a QVT podem elevar o nível de comprometimento e qualidade e diminuir os índices de turnover, mas não aumentam, necessariamente, a produtividade e a satisfação, uma vez que não constituem uma relação direta de causa-e-efeito. Assim, os tipos de atividades de QVT compreendem: i) resolução participativa de problemas; ii) reestruturação da natureza do trabalho; iii) sistemas inovadores de recompensas; iv) melhorias no ambiente de trabalho (N ADLER; LAWLER III, 1983).

Ainda nesse sentido, as definições de QVT envolvem uma dimensão variada de aspectos, relacionados com a satisfação e envolvimento no trabalho, motivação, produtividade, saúde e segurança no ambiente laboral, desenvolvimento de competências e equilíbrio entre vida familiar e trabalho (SHAHBAZI; SHOKRZADEH; BEJANI; MALEKINIA; GHORONEH, 2011); e com o bem-estar no trabalho, sistemas de recompensa e gestão do desempenho (YEO; LI, 2012).

Greenhaus, Collins e Shaw (2003) propõem três componentes em relação ao equilíbrio da vida familiar e trabalho: equilíbrio de tempo, de envolvimento e de satisfação, podendo, estes, a depender dos seus níveis, apresentar um equilíbrio positivo ou negativo. Os referidos autores acreditam que um indivíduo somente atinge o equilíbrio entre as duas esferas quando se engaja ou está igualmente satisfeito em ambas.

Aprofundar a investigação da GT relacionada a QVT leva em consideração o contexto contemporâneo de forte competição, cons- 
tantes mudanças na tecnologia e inovação (DE PAOLA; GIOIA, 2016), exigências e retornos imediatos, no qual estar apressado e apagar incêndios com frequência são consideradas características de comprometimento, diligência e zelo pelo interesse comum (MYSZEWSKI, 2015).

Dessa forma, o tempo se tornou um ativo importante e de valor, e o ritmo e a imprevisibilidade das mudanças tendem a delimitar o tempo do processo de decisão, fazendo com que o estado de pressa se torne natural (MYSZEWSKI, 2015). A limitação e a pressão de tempo envolvidas nas decisões e na resolução de tarefas podem elevar os níveis de estresse e afetar o desempenho dos indivíduos, levando-os, inclusive, a terem maior dificuldade em identificar informações importantes dentre tantas outras urgentes entendidas como essenciais (DE PAOLA; GIOIA, 2016).

\subsection{Gestão do tempo}

A constante busca pela melhor administração do tempo tem sido uma preocupação para realização pessoal e profissional e não poderia ser diferente, pois como dissertou Drucker (1967), tudo requer tempo, e esta é a única condição verdadeiramente universal, pois todo trabalho ocorre em tempo e consome tempo.

A GT pode ser descrita como uma forma autorregulada e subjetiva de tentar usar o tempo, eficientemente, para o alcance de resultados (KOCH; KLEINMANN, 2002), facilitando a rotina e a organização das tarefas.

Claessens et al. (2007) relatam que o termo gestão de tempo é enganoso, e, estritamente falando, o tempo não pode ser gerenciado, porque é um fator inacessível. Apenas a forma como a pessoa lida com o tempo pode ser alterada, corroborando a ideia de que fatores elevados de estresse e tensão podem levar a uma má administração do tempo, impactando diretamente na produtividade individual ou pessoal.

A maneira como o indivíduo administra a GT está diretamente relacionada com o meio no qual está inserido e com a intensa rotina de trabalho. A carga de trabalho elevada e a baixa autonomia tem um efeito principal significativo em relação ao estresse (PARKES; MENDHAN; VON RABENAU, 1994; BARNETT; BRENNAN, 1997). 
Lee e Liebenau (2002) comentam que o taylorismo defendeu a eficiência na gestão e produtividade através do tempo e planejamento. O planejamento é a chave para a eficiência do processo de modo geral, o que reflete significativamente em uma gestão do tempo equilibrada e sensata (MYSZEWSKI, 2015).

Barbosa (2011) faz um panorama do impacto da vida atarefada na saúde e nos relacionamentos interpessoais, comentando que o ritmo desenfreado em que se vive atualmente leva o estresse e o constante resolver urgências a padrões corriqueiros, considerados normais (MYSZEWSKI, 2015). A busca pelo maior equilíbrio entre os diversos papeis sociais requer mudanças pessoais e capacidade de fazer escolhas entre o que é importante, urgente e circunstancial (BARBOSA, 2011).

A ideia de escolha e planejamento como competências essenciais a uma boa GT pode ser amparada no modelo de Walton (1973) de análise dos elementos que compõem a QVT. Uma das oito categorias conceituais envolve a dedicação ao trabalho em detrimento das outras esferas da vida pessoal. Assim, encontrar o equilíbrio entre os vários papeis requer o uso adequado de tempo e energia para que o indivíduo possa voltar a sua atenção a outras atividades sociais ou familiares.

Levando em consideração que o trabalho, cada vez mais, exige o desenvolvimento de múltiplas tarefas em curto espaço de tempo, é interessante que as empresas passem a investigar como as pessoas dividem seu tempo para o alcance das metas propostas, e quais são os efeitos dessa variedade paralela de atividades na vida e no trabalho dos indivíduos (SEHRISH; ZUBAIR, 2013).

Kaufman-Scarborough e Lindquist (1999), aprofundaram os estudos iniciais sobre a GT - que já incluíam a necessidade de priorização, criação e uso de listas, e horários para cada atividade - relacionando os comportamentos da GT com a possibilidade de realização das atividades de cada indivíduo.

Nesse contexto, a policronicidade foi tratada como a capacidade da pessoa de executar duas ou mais tarefas ao mesmo tempo (BLUEDORN; KAUFMAN; LANE, 1992; SLOCOMBE; BLUEDORN, 1999), ao invés de se concentrar em apenas uma (POPOSKI; OSWALD; CHEN, 2008). A monocronicidade envolve 
a opção da pessoa por uma vida mais organizada, realizando uma tarefa por vez, a partir de programações detalhadas (MADJAR; OLDHAM, 2006).

O perfil das pessoas afetadas pela policronicidade ou monocronicidade está diretamente relacionado com a forma como elas lidam com a gestão do tempo no trabalho (MADJAR; OLDHAM, 2006). Kaufman-Scarborough e Lindquist (1999) reforçam que essa diferença de estilo pode coexistir dentro das organizações, sendo provável fonte de conflito dada a sua contrastante abordagem do tempo e do cumprimento de metas.

Por sua vez, Sehrish e Zubair (2013) consideram a relação GT e policronicidade de suma importância para as organizações, uma vez que a QVT pode ser influenciada pelos dois fatores, estando em proporção direta com os resultados obtidos tanto individualmente como em termos organizacionais.

Sehrish e Zubair (2013) enfatizam ainda que o mais importante na GT é como utilizar eficientemente o tempo disponível, evitando um problema comum mencionado por Koch e Kleinmann (2002) que é a negligência de atividades importantes pelo fato delas não serem urgentes.

\subsection{Modelos de gerenciamento do tempo}

A importância cada vez maior do tempo é refletida tanto na teoria, como em publicações práticas (CLAESSENS et al., 2007). Questionamentos e discussões a respeito de como gerir o tempo no trabalho existem desde a década de 1950 e vários autores propõem métodos a respeito dessa questão (MCCAY, 1959; DRUKER, 1967; MACKENZIE, 1972; LAKEIN, 1973; RICHARDS, 1987) objetivando o aumento do desempenho (DAVIS, 2000; VAN EERDE, 2003; H ̈̈FNER; STOCK; PINNEKER; STROHLE, 2015) e do bem-estar (MACAN; SHAHANI; DIPBOYE; PHILLIPS, 1990; BRITTON; TESSER, 1991), pois estes são os principais resultados tanto para indivíduos como para as empresas (AEON; AGUINIS, 2017). Essa ênfase na administração do tempo decorre da crença popular não comprovada de que a má alocação de tempo não apenas aumenta o estresse do trabalhador, mas também prejudica o desempenho (MACAN, 1994). 
De acordo com Lakein (1973), no gerenciamento do tempo, primeiro os indivíduos determinam suas necessidades e desejos e então os classificam em termos de importância (MACAN, 1994). Macan et al. (1990) apontam três fatores relacionados à gestão do tempo que são consistentes com os propostos por Lakein (1972): definição de metas e prioridades, fazer listas de atividades a serem realizadas, e a questão de escolha da organização a trabalhar (MACAN, 1996; BURRUS; JACKSON; HOLTZMAN; ROBERTS, 2017).

Mesmo com toda a atenção voltada para a GT, poucas investigações foram conduzidas sobre os processos de uso do tempo de forma eficaz (usando horário nobre para a realização de tarefas importantes) e completar o trabalho dentro de prazos (CLAESSENS et al., 2007).

Os estudos envolvendo a temática têm utilizado principalmente questionários de auto relato destinados a medir os comportamentos de tempo, sendo três deles utilizados mais frequentemente: i)Escala de Comportamento de Gestão do Tempo (TMBS) (MACAN et al., 1990); ii) Questionário de Estrutura do Tempo (TQS) (BOND; FEATHER, 1988); e, iii)Questionário de Gestão do Tempo (TQM) (BRITTON; TESSER, 1991).

O TMBS foi construído por Macan et al. (1990) baseado em uma listagem de conceitos de comportamento de gerenciamento do tempo, tendo como construtos o estabelecimento de metas e prioridades, os mecanismos de gerência do tempo, a ordenação de trabalho e o controle percebido do tempo. O TSQ foi formulado utilizando seis fatores, mas apenas cinco foram nomeados: sentido de propósito, rotina estruturada, orientação presente, organização eficaz e persistência (BOND; FEATHER, 1988). Já o Modelo TMQ inclui itens relacionados a atitudes e planejamento sobre a alocação do tempo e está pautado em três fatores: planejamento de curto e longo prazo, e atitudes.

Um ponto em comum a se observar nos três instrumentos é o comportamento planejado, voltado para o desenvolvimento de tarefas e atividades de planejamento. Quanto à associação entre o comportamento de gestão de tempo e resultados, percebeu-se que o planejamento apresentou os resultados mais significativos. Bond e Feather (1988) constataram que o fator sentido de propósito obteve 
a maior pontuação total e foi considerado o mais importante do TSQ. Macan (1990) evidenciou que o estabelecimento e priorização de metas estava significativamente relacionada com os resultados. Britton e Tesse (1991) encontraram uma relação positiva entre o planejamento de curto prazo e os resultados, enquanto que o de longo prazo não estava relacionado.

Além desses modelos, foi encontrado ainda o Modelo Australiano de Organização e Gestão do Tempo (ATOMS) proposto por Roberts, Krause e Suk-Lee (2016) composto por seis fatores: senso de propósito, cumprimento de prazos, mecanismos de gestão do tempo, lidar com o fluxo circunstancial, planejamento e organização.

Outras variáveis concernentes à gestão do tempo foram propostas e estudadas por diversos autores. Francis-Smythe e Robertson (1999) inseriram o fator personalidade, relacionado à pontualidade, impaciência e policronicidade. Kaufman-Scarborough e Lindquist (1999) perceberam a diferença entre a gestão do tempo e os estilos monocrômico e policrônico: esse segundo estilo consiste em pessoas mais capazes de gerenciar interrupções de trabalho, realizando atividades de urgência mais rápido do que as monocrômicas. Além disso, avaliaram a importância relativa das atividades através de um plano de prioridades. Van Eerde (2003) e Häfner et al. (2014) estudaram a relação entre a procrastinação e a gestão do tempo.

Bird e Jordan (1987) mediram os pensamentos e sentimentos de uma pessoa; outros estudos investigaram os efeitos dos resultados comportamentais relacionados ao estresse (MACAN, 1994; AD AMS; JEX, 1999; D AVIS, 2000); satisfação no trabalho (MACAN, 1994; D AVIS, 2000); sobrecarga de trabalho (BURT; KEMP, 1994); tensão e pressão (MACAN, 1994; D AVIS, 2000); exaustão emocional (PEETERS; RUTTE, 2005); e saúde (BOND; FEATHER, 1988).

Landy, Rastegary e Thayer (1991) desenvolveram a Escala de Urgência de Tempo, relacionando tempo e medidas de urgência, satisfação no trabalho e estresse. Alay e Kocak (2002) desenvolveram um modelo ajustado do TMBS (MACAN et al., 1990), baseado numa análise fatorial dividindo em três construtos: planejamento, atitudes e desperdício.

Para melhor gerir o tempo, Cannon (1996) afirma que os indivíduos devem criar estratégias, ou seja, elaborar um plano para 
autorregulação e desenvolvimento de habilidades voltado para o tempo. A abordagem de Covey (2014) afirma que o desafio é gerenciar a nós mesmos e não o tempo. Seu método é baseado em como organizar e executar prioridades (MACAN, 1990; KAUFMAN-SCARBOROUGH; LINDQUIST, 1999), utilizando a matriz de gerenciamento de tempo, na qual as atividades podem ser definidas por seu grau de urgência (MACAN, 1990) e não urgência, pela sua exigência ou não de atenção imediata, e pela sua importância (MACAN, 1990), que estão relacionadas ou não aos objetivos e valores pessoais (MACAN, 1990; BARBOSA, 2011).

Myszewski (2015) aborda a GT relacionando-o à pressa e situações de emergência. As circunstâncias são tratadas como excepcional, associando o não cumprimento de atividades em cima da hora com perigo para a organização e, por conseguinte, para todos os seus membros.

No Brasil, Barbosa (2011) desenvolveu o modelo chamado de Tríade do Tempo, tendo como construtos urgência, importância e circunstância. Este último, também é chamado de desperdício por Landy et al. (1991) e de tarefas não importantes e não urgentes por Covey (2014).

\section{Metodologia}

Este estudo é caracterizado como pesquisa quantitativa de natureza descritiva, por meio de uma survey aplicada a trabalhadores de diferentes ramos de atividades no Nordeste do Brasil, para analisar a influência da gestão do tempo sobre a qualidade de vida no trabalho.

Conforme Creswell e Creswell (2017) e Richardson (2017), a pesquisa quantitativa utiliza a quantificação tanto na coleta como no tratamento das informações geradas pelo uso de técnicas estatísticas, tendo como principal objetivo mostrar resultados com maior exatidão, evitando distorções de análises e de interpretação, assim como também garante uma margem de segurança maior quanto às interferências. A natureza descritiva permitiu investigar a relação entre as variáveis e construtos do modelo utilizado para medir a GT através de um processo estruturado de coleta e análise de dados (COOPER; SCHINDLER, 2016). 
Neste cenário, assume-se como um levantamento do tipo survey um conjunto de centenas ou milhares de elementos (MALHOTRA, 2012). Coerentemente, em virtude de uma amostra significativa, o instrumento de pesquisa quantitativa aconteceu por meio de uma Survey descritiva. A pesquisa foi realizada junto a gestores, técnicos administrativos e vendedores nos ramos de atividade da educação, comércio varejista, tecnologia e saúde no Nordeste do Brasil, tendo 454 pesquisados como amostra não probabilística por conveniência (MALHOTRA, 2012). A coleta de dados foi realizada entre 2017 e 2018 por e-mail, utilizando um formulário eletrônico do Google Docs.

O questionário possui 21 questões, das quais 03 foram relacionadas a dados demográficos (sexo, função e ramo de atividade da empresa na qual trabalha), e 18 são afirmações utilizando a escala Likert de 5 pontos, sendo 1 para nunca e 5 para sempre. As afirmações foram divididas em 03 blocos: o primeiro abordando a percepção dos indivíduos sobre desperdício/circunstâncias temporais de vida pessoal e de trabalho; no segundo foram avaliadas as atividades consideradas importantes; e o terceiro mediu o nível em que os indivíduos desenvolvem atividades que são consideradas como urgentes.

Os três construtos utilizados na pesquisa foram adaptados de estudos anteriores, conforme Tabela 1.

Para analisar a confiabilidade e normalidade dos dados, foram aplicados testes estatísticos (HAIR JR. et al., 2006; MALHOTRA, 2012): teste de normalidade de Kolmogorov-Smirnov (KS) e Shapiro-Wilk (SW); confiabilidade, Alpha de Cronbach, e Kaiser-Meyer-Olkin (KMO); e teste de esferacidade de Bartlett, que possibilita averiguar as correlações entre as variáveis. Foi utilizado o software SPSS Statistcs (versão 21) para a realização das análises, mantendo o alinhamento de aplicação da análise fatorial confirmatória (AFC) nos estudos de Hair Jr. et al. (2006).

Na análise dos dados foi utilizada a estatística descritiva, a AFC, a correlação de Pearson e a regressão linear múltipla (RLM). De acordo com Brown (2014), a AFC é entendida como uma técnica confirmatória, uma vez que é utilizada para avaliar hipóteses de formação de construtos em pesquisas confirmatórias. No desenvolvimento da AFC, partiu-se para a realização de análise intrabloco, com a avaliação das cargas fatoriais, comunalidade e variância explicada. 
Tabela 1: Resumo dos construtos e respectivas referências

\begin{tabular}{|c|c|c|}
\hline Constructo & Descrição & Referências \\
\hline $\begin{array}{l}\text { Circunstân- } \\
\text { cia/ Desper- } \\
\text { dício (CIR) }\end{array}$ & $\begin{array}{l}\text { Está voltada para qual- } \\
\text { quer tipo de atividade } \\
\text { que a pessoa é levada a } \\
\text { fazer em função da situa- } \\
\text { ção, condição ou ambien- } \\
\text { te, fazendo-a desfocar do } \\
\text { que é importante e/ou } \\
\text { urgente. }\end{array}$ & $\begin{array}{l}\text { Lakein (1972); Bond e Feather } \\
\text { (1988); Macan et al. (1990); } \\
\text { Britton e Tesser (1991); Landy } \\
\text { et al. (1991); Alay e Kocak } \\
\text { (2002); Barbosa (2011); Covey } \\
\text { (2014); Myszewski (2015); e } \\
\text { Roberts, Krause, e Suk-Lee } \\
\text { (2016). }\end{array}$ \\
\hline $\begin{array}{l}\text { Importân- } \\
\text { cia (IMP) }\end{array}$ & $\begin{array}{l}\text { Abrange todas as ati- } \\
\text { vidades que são verda- } \\
\text { deiramente importantes } \\
\text { na vida do indivíduo, } \\
\text { que trazem resultados } \\
\text { mais efetivos, que fazem } \\
\text { diferença para ele, para } \\
\text { o trabalho, para a comu- } \\
\text { nidade e para as pessoas } \\
\text { com quem convive. }\end{array}$ & $\begin{array}{l}\text { Macan et al. (1990); Landy } \\
\text { et al. (1991); Claessens et al. } \\
\text { (2004); Barbosa (2011); e Co- } \\
\text { vey (2014). }\end{array}$ \\
\hline $\begin{array}{l}\text { Urgência } \\
\text { (URG) }\end{array}$ & $\begin{array}{l}\text { São decisões relacionadas } \\
\text { às tarefas que devem ser } \\
\text { realizadas de imediato, e } \\
\text { que podem gerar algum } \\
\text { tipo de problema se não } \\
\text { forem executadas. }\end{array}$ & $\begin{array}{l}\text { Bond e Feather (1988); Macan } \\
\text { (1990); Francis-Smythe e Ro- } \\
\text { bertson (1999); Kaufman-Scar- } \\
\text { borough e Lindquist (1999); } \\
\text { Alay e Kocak (2002); Van } \\
\text { Eerde (2003); Barbosa (2011); } \\
\text { Käser et al. (2013); e Covey } \\
\text { (2014). }\end{array}$ \\
\hline
\end{tabular}

Fonte: Elaborado pelos autores (2019)

Segundo Eisinga, Te Grotenhuis e Pelzer (2013) a correlação de Pearson comprova o quanto uma variável está correlacionada com outra, mostrando o nível de interação entre as variáveis de um constructo. Já a RLM procura estabelecer a associação entre variáveis, determinando suas interações nos constructos (HAIR JR. et al., 2006). 


\section{RESULTADO E DISCUSSÃo}

Os questionários foram aplicados junto a 454 indivíduos, divididos em cargos: 287 (63,2\%) gestores, 143 técnicos (32,5\%) e 24 vendedores (5,3\%). Do total de respondentes, 260 são do sexo feminino, representando $57,3 \%$, e 194 do sexo masculino $(42,7 \%)$, distribuídos nos seguintes ramos de atividade: educação $(11,67 \%)$, tecnologia $(25,55 \%)$, comércio $(23,79 \%)$ e saúde $(38,99 \%)$.

\subsection{Resultados da análise fatorial confirmatória}

Inicialmente foram realizados os testes de normalidade e confiabilidade dos dados. O teste de KS e de SW apresentaram resultados significativos, uma vez que $\mathrm{p}>0,05$, mostrando a normalidade dos dados. Os resultados referentes ao teste de Alpha de Cronbach das variáveis (Tabela 2) revelaram valores de 0,645. Segundo Hair Jr. et al. (2006), para que os constructos sejam consistentes e apropriados, o valor deve ser maior que 0,8. Entretanto, para estudos nas áreas sociais, é indicado o mínimo de 0,6 (DEVELLIS, 1991; FIELD; MILES; FIELD, 2012), possibilitando validar a robustez do instrumento, pois a pesquisa é enquadrada nas ciências sociais. Os testes de KMO e Esferacidade de Bartlett (Tabela 2) revelaram valores de 0,693 (próximo a 0,7) e nível de significância de 0,00 (valor inferior a 0,05), demonstrando a existência de correlação entre as variáveis, respectivamente. Também foi possível verificar a comunalidade entre as questões, observando que duas questões referente ao constructo Circunstância/Desperdício possuem valores abaixo de 0,5, assim como o constructo Importância e Urgência. Optou-se em não retirar as questões da análise, uma vez que se trata de um questionário com variáveis em número reduzido, no qual os construtos possuem grande relação si.

Desse modo, observa-se que, de acordo com as recomendações de Malhotra (2012) e Hair Jr. et al. (2006) e os testes de normalidade e confiabilidade indicam que os dados são adequados para a aplicação da análise fatorial confirmatória. 
Tabela 2: Resultado Alpha de Cronbach, KMO

$e$ Teste de Esferacidade de Bartlett

\begin{tabular}{lll}
\hline Teste & Valor encontrado \\
\hline Alpha de Cronbach & 0,645 \\
\hline KMO & 0,693 \\
\hline \multirow{2}{*}{$\begin{array}{l}\text { Teste de Esferacidade } \\
\text { de Bartlett }\end{array}$} & Qui-quadrado & $1,180,057$ \\
\cline { 2 - 3 } & Significância & $0,000^{*}$ \\
\hline
\end{tabular}

* Nível de significância $\mathrm{p}<0,001$

Fonte: Elaborado pelos autores (2019)

Dando continuidade, foi realizada a análise de Pearson para verificar a multicolinearidade, analisando se as variáveis possuem correlação maior que 0,8 (Hair Jr. et al., 2006), dividindo tal análise em três blocos (constructos), sendo eles: i) Circunstância/Desperdício; ii) Importância; e, iii) Urgência.

\subsection{Resultado da análise fatorial intrabloco}

A análise intrabloco tem como maior objetivo verificar o grau de associação entre os fatores de cada bloco e o quanto se representam num conceito único (HAIR JR. et al., 2006). Diante dessa questão, foi realizada a análise dos constructos, examinando o conjunto de variáveis observadas dentro de cada bloco, a comunalidade, as cargas fatoriais, o KMO, o teste de Esferacidade de Bartlett e a variância explicada.

O estudo propõe a divisão em três blocos: i) Bloco I - Circunstância/Desperdício; ii) Bloco II - Importância; e, iii) Bloco III - Urgência.

A Tabela 3 demonstra a validação dos dados para a análise intrabloco do Bloco I - Circunstância/Desperdício. 
Tabela 3: Validação dos dados para o Bloco I - Circunstância/Desperdício

\begin{tabular}{ll}
\hline Teste & Valor encontrado \\
\hline KMO & 0,599 \\
\hline Teste de Esferacidade de Bartlett & $112,188^{*}$ \\
\hline Variância Explicada $\quad 45,4 \%$ \\
\hline \multicolumn{2}{c}{${ }^{*}$ Nível de significância p<0,001 } \\
Fonte: Elaborado pelos autores (2019)
\end{tabular}

Os valores referentes ao KMO é de 0,599 , e chegam bem próximos do que é considerado satisfatório $(0,50)$ para Hair Jr. et al. (2006). O teste de Esferacidade de Bartlett são aceitáveis, (MALHOTRA, 2012) e a variância explicada é de $45,44 \%$.

Posteriormente, foi verificado os valores de comunalidade e cargas fatoriais das variáveis do Bloco I (Tabela 4), que, de acordo com Hair Jr. et al. (2006) deverão ser iguais ou maiores que 0,5, alertando para possíveis exclusões de variáveis, conforme escolha ou necessidade do pesquisador.

Todas as variáveis apresentaram carga fatorial acima de 0,5, conforme recomendado por Hair Jr. et al. (2006). Esse resultado corrobora as pesquisas de Lakein (1972), Bond e Feather (1988), Macan et al. (1990), Landy et al. (1991), Alay e Kocak (2002), Barbosa (2011), Covey (2014), Myszewski (2015) e Roberts, Krause e Suk-Lee (2016) que destacam o quanto questões relacionadas a maneiras de desperdício de tempo são consideradas relevantes, sendo enunciadas por muitos autores que abordam a temática de gestão do tempo.

Em relação à comunalidade, os valores apresentados foram inferiores a 0,5, seguindo recomendação de Hair Jr. et al. (2006) e Field, Miles e Field, 2012, com a exceção da questão CIR5 que obteve comunalidade de 0,612, revelando grande correlação dessa variável com as outras. No entanto, os resultados das demais questões indicam que não há uma grande correlação entre as variáveis observáveis, mas elas foram mantidas devido à necessidade de alinhamento das questões com o objetivo da pesquisa. 
Tabela 4: Análise Fatorial intrabloco

- Bloco I - Circunstância/Desperdício

\begin{tabular}{|c|c|c|c|}
\hline Bloco & Variáveis Observáveis & Comunalidade & $\begin{array}{l}\text { Cargas } \\
\text { Fatoriais }\end{array}$ \\
\hline \multirow{6}{*}{$\begin{array}{l}\text { Circunstân- } \\
\text { cia/Desper- } \\
\text { dício }\end{array}$} & $\begin{array}{l}\text { CIR1- Costumo ir a eventos, } \\
\text { festa ou cursos, mesmo sem ter } \\
\text { muita vontade, para agradar ao } \\
\text { chefe, aos amigos ou à família. }\end{array}$ & 0,318 & 0,525 \\
\hline & $\begin{array}{l}\text { CIR2- Quando recebo um novo } \\
\text { e-mail costumo dar uma olhada } \\
\text { para checar o conteúdo. }\end{array}$ & 0,572 & 0,709 \\
\hline & $\begin{array}{l}\text { CIR3- Assumo compromissos } \\
\text { com outras pessoas ou aceito } \\
\text { novas posições na empresa, } \\
\text { mesmo que não goste muito } \\
\text { da nova atividade, se for para } \\
\text { aumentar meus rendimentos ou } \\
\text { obter uma promoção. }\end{array}$ & 0,434 & 0,647 \\
\hline & $\begin{array}{l}\text { CIR4- Nos dias de descanso, } \\
\text { costumo passar boa parte do } \\
\text { dia assistindo à televisão, jogan- } \\
\text { do ou acessando a internet. }\end{array}$ & 0,612 & 0,673 \\
\hline & $\begin{array}{l}\text { CIR5- Costumo participar de } \\
\text { reuniões sem saber direito o } \\
\text { conteúdo, o porquê da minha } \\
\text { presença ali ou a que resultado } \\
\text { aquele encontro pode levar. }\end{array}$ & 0,481 & 0,642 \\
\hline & $\begin{array}{l}\text { CIR6- Leio muitos e-mails } \\
\text { desnecessários, com piadas, } \\
\text { correntes, propagandas, apre- } \\
\text { sentações, produtos, etc. }\end{array}$ & 0,310 & 0,524 \\
\hline
\end{tabular}

Fonte: Elaborado pelos autores (2019)

A Tabela 5 apresenta a validação dos dados para a análise intrabloco do Bloco II - Importância, indicando KMO e o teste de esferacidade de Bartlett como aceitáveis, e a variância explicada de $56,89 \%$. 
Tabela 5: Validação dos dados para o Bloco II - Importância

\begin{tabular}{ll}
\hline Teste & Valor encontrado \\
\hline KMO & 0,665 \\
\hline Teste de Esferacidade de Bartlett & $409,296^{*}$ \\
\hline Variância Explicada & $56,9 \%$ \\
\hline
\end{tabular}

* Nível de significância p<0,001

Fonte: Elaborado pelos autores (2019)

A Tabela 6 demonstra a análise intrabloco do Bloco II, evidenciando apenas a questão IMP7 $(0,446)$ com carga fatorial abaixo do recomendado. A questão é relativa à frequência com que as pessoas costumam visitar outras que são relevantes em sua vida, de forma que, considerando a importância teórica para a explicação do constructo, decidiu-se manter a variável na pesquisa. A comunalidade nas questões IMP7 $(0,389)$ e IMP10 $(0,408)$ foi abaixo de 0,5 , mas optou-se em mantê-las, considerando a sua relevância para a formação e entendimento do constructo.

A afirmativa IMP9, que aborda o planejamento por escrito das atividades semanais obteve carga fatorial de 0,698, tendo respaldo nos estudos de Myszewski (2015), que afirma que o planejamento é a chave para a eficiência do processo, refletindo diretamente na gestão do tempo equilibrada e sensata. Lee e Liebenau (2002) afirmam que o planejamento consiste numa tentativa de reduzir a quantidade de tempo que se leva para completar uma determinada quantidade de trabalho. O modelo sugerido por Bond e Feather (1988) inclui itens relacionados ao planejamento da alocação do tempo, pautado em três fatores: planejamento de curto e longo prazo, e atitudes. Além disso, competências essenciais como planejamento para uma boa gestão do tempo podem ser amparadas no modelo de Walton (1973) de análise dos elementos que compõem a QVT.

A Tabela 7 confirma a validação dos dados para a análise intrabloco III - Urgência, revelando o KMO e teste de esferacidade de Bartlett aceitáveis e variância explicada de 51,10\%. 
Tabela 6: Análise Fatorial intrabloco - Bloco II - Importância

\begin{tabular}{|c|c|c|c|}
\hline Bloco & Variáveis Observáveis & $\begin{array}{l}\text { Comunali- } \\
\text { dade }\end{array}$ & $\begin{array}{l}\text { Cargas } \\
\text { Fatoriais }\end{array}$ \\
\hline \multirow{6}{*}{ Importância } & $\begin{array}{l}\text { IMP7- Costumo visitar com } \\
\text { regularidade pessoas relevantes } \\
\text { em minha - amigos, parentes, } \\
\text { filhos. }\end{array}$ & 0,389 & 0,446 \\
\hline & $\begin{array}{l}\text { IMP8- Tenho um tempo definido } \\
\text { para dedicar a mim mesmo e } \\
\text { nele, posso fazer o que quiser. }\end{array}$ & 0,598 & 0,642 \\
\hline & $\begin{array}{l}\text { IMP9- Faço um planejamento } \\
\text { por escrito de tudo o que preciso } \\
\text { fazer durante a semana. }\end{array}$ & 0,761 & 0,698 \\
\hline & $\begin{array}{l}\text { IMP10- Posso afirmar que estou } \\
\text { conseguindo realizar tudo o que } \\
\text { gostaria em minha vida e que o } \\
\text { tempo está passando na veloci- } \\
\text { dade correta. }\end{array}$ & 0,408 & 0,639 \\
\hline & $\begin{array}{l}\text { IMP11- Quando quero alguma } \\
\text { coisa, defino esse objetivo por } \\
\text { escrito, estabeleço prazos em mi- } \\
\text { nha agenda, monitoro os resulta- } \\
\text { dos obtidos e os comparo com os } \\
\text { esperados. }\end{array}$ & 0,747 & 0,661 \\
\hline & $\begin{array}{l}\text { IMP12- Faço exercícios com } \\
\text { regularidade, alimento-me da } \\
\text { forma adequada e desfruto horas } \\
\text { suficientes de lazer. }\end{array}$ & 0,510 & 0,529 \\
\hline
\end{tabular}

Fonte: Elaborado pelos autores (2019)

Tabela 7: Validação dos dados para o Bloco III - Urgência

\begin{tabular}{ll}
\hline Teste & Valor encontrado \\
\hline KMO & 0,662 \\
\hline Teste de Esferacidade de Bartlett & $282,998^{*}$ \\
\hline Variância Explicada & $51,1 \%$ \\
\hline
\end{tabular}

* Nível de significância $p<0,001$

Fonte: Elaborado pelos autores (2019) 
A Tabela 8 mostra a análise intrabloco do Bloco III - Urgência, demonstrando cargas fatoriais aceitáveis para validação da escala, maior ou igual a 0,5, exceto as questões URG16 e URG18, que foram mantidas no modelo devido à sua importância para a composição e compreensão do constructo.

As variáveis URG16, URG17 e URG18 apresentaram comunalidade inferior a 0,5, mas optou-se em mantê-las para a análise da RLM, pois ajudam a compor o constructo.

Tabela 8: Análise Fatorial intrabloco - Bloco III - Urgência

\begin{tabular}{clll}
\hline \multirow{2}{*}{ Bloco } & $\begin{array}{l}\text { Variáveis Observáveis } \\
\text { URG13- Não consigo realizar }\end{array}$ & $\begin{array}{l}\text { Comunali- } \\
\text { dade }\end{array}$ & $\begin{array}{l}\text { Cargas } \\
\text { Fatoriais }\end{array}$ \\
\hline $\begin{array}{l}\text { tudo que me propus a fazer no } \\
\text { dia e preciso cumprir hora extra } \\
\text { e levar trabalho para casa. }\end{array}$ & 0,612 & 0,726 \\
$\begin{array}{l}\text { URG14- É comum aparecerem } \\
\text { problemas inesperados no meu } \\
\text { dia a dia. }\end{array}$ & 0,691 & 0,756 \\
$\begin{array}{l}\text { URG15- Consigo melhores resul- } \\
\text { tados e me sinto mais produtivo } \\
\text { quando estou sob pressão ou } \\
\text { quando o prazo é curto. }\end{array}$ & 0,632 & 0,654 \\
$\begin{array}{l}\text { URG16- Costumo deixar para fa- } \\
\text { Urgência relatórios, imposto de renda, } \\
\text { compras de natal, estudar para } \\
\text { provas e outras tarefas perto do } \\
\text { prazo de entrega. }\end{array}$ & 0,281 & 0,425 \\
$\begin{array}{l}\text { URG17- Estive atrasado com } \\
\text { minhas tarefas ou reuniões nas } \\
\text { últimas semanas. }\end{array}$ & 0,485 \\
$\begin{array}{l}\text { URG18- É comum reduzir meu } \\
\text { horário de almoço ou até mesmo } \\
\text { comer enquanto trabalho para } \\
\text { concluir um projeto ou tarefa. }\end{array}$ & 0,366 \\
\hline
\end{tabular}

Fonte: Elaborado pelos autores (2019)

No Bloco III merece destaque a variável URG14 (É comum aparecerem problemas inesperados no meu dia a dia), tendo a maior 
carga fatorial $(0,726)$. A questão está associada ao surgimento de tarefas urgentes que são consideradas prioritárias e que devem ser realizadas imediatamente (MACAN, 1990; KA UFMAN-SCARBOROUGH; LINDQUIST, 1999; B ARBOSA, 2011). Para Myszewski (2015) o estado de pressa está se tornando natural, fazendo com que as pessoas cada vez mais detenham-se em atividades urgentes, tendo dificuldade em equilibrar vida familiar e trabalho (SHAHBAZI et al., 2011).

A variável que apresentou a menor carga fatorial foi URG16 (Costumo deixar para fazer relatórios, imposto de renda, compras de natal, estudar para provas e outras tarefas perto do prazo de entrega), com 0,425 , indicando que ela pouco contribui para a formação do construto, uma vez que detalha situações que podem ser planejadas, distanciando-a de outras variáveis que priorizavam situações inesperadas e/ou com os prazos vencidos.

\subsection{Resultados da regressão linear múltipla}

A análise de regressão linear múltipla foi realizada dentro dos blocos e utilizando a questão de fechamento relacionada a QVT, para a qual os indivíduos atribuíram nota de 1 a 10 para expressarem como percebem a sua qualidade de vida. Tal questão foi relacionada aos constructos de gestão do tempo.

A Tabela 9 expõe a primeira análise tendo a MedIMP - Média das questões de Importância (IMP1, IMP2, IMP3, IMP4, IMP5 e IMP6) como variável dependente e o restante das questões dos constructos circunstância/desperdício e urgência (CIR1, CIR2, CIR3, CIR4, CIR5, CIR6, URG1, URG2, URG3, URG4, URG5 e URG6) como variável independente.

Tabela 9: Análise de regressão linear múltipla para MedIMP e Bloco I e III

\begin{tabular}{lllll}
\hline Modelo & $\mathrm{R}$ & $\mathrm{R}^{2}$ & $\mathrm{R}^{2}$ Ajustado & Std Erro Estimado \\
\hline 1 & $0,379^{\mathrm{a}}$ & 0,144 & 0,120 & 0,63973 \\
\hline
\end{tabular}

a. Predictors: (Constant), URG6, CIR2, CIR3, URG2, CIR6, CIR4, URG4, CIR1, URG5, CIR5, URG3, URG1

b. Dependent Variable: Média de Importante

Fonte: Elaborado pelos autores (2019) 
Os dados obtidos da análise apontam que as variáveis do Bloco I e do Bloco III explicam apenas 14,4\% a variável dependente, justificando-se pelos estudos de Barbosa (2011) e Covey (2014), uma vez que explicam a importância como sendo correspondente à capacidade de se dedicar às atividades que podem ser programadas e que geram valor ao indivíduo, aproveitando melhor o tempo quando não se volta às circunstâncias/desperdícios e urgências.

A Tabela 10 apresenta os resultados da segunda regressão linear gerada, tendo URG1 (Não consigo realizar tudo que me propus a fazer no dia e preciso cumprir hora extra e levar trabalho para casa) como variável dependente, e o restante do Bloco III (URG2, URG3, URG4, URG5 e URG6) como variáveis independentes.

Tabela 10: Análise de regressão linear múltipla para Bloco III

\begin{tabular}{lllll}
\hline Modelo & $\mathbf{R}$ & $\mathbf{R}^{\mathbf{2}}$ & $\mathbf{R}^{\mathbf{2}}$ Ajustado & Std Erro Estimado \\
\hline 1 & $0,519^{\mathrm{a}}$ & 0,270 & 0,261 & 0,82820 \\
\hline
\end{tabular}

Fonte: Elaborado pelos autores (2019)

Os dados apresentados revelam que as variáveis independentes respondem em $27 \%$ a variável dependente, validando assim a urgência como constructo utilizado na gestão do tempo (FRANCIS-SMYTHE; ROBERTSON, 1999; KAUFMAN-SCARBOROUGH; LINDQUIST, 1999; ALAY E KOCAK, 2002; VAN EERDE, 2003; BARBOSA, 2011; KÄSER et al., 2013; COVEY, 2014).

A Tabela 11 demonstra a última análise da regressão linear múltipla, tendo a questão de fechamento "Qual nota você atribui para a sua qualidade de vida" como variável dependente e os Blocos I, II e III como variáveis independentes.

Tabela 11: Análise de regressão linear múltipla a questão de fechamento e os Blocos I, II e III

\begin{tabular}{lllll}
\hline Modelo & $\mathbf{R}$ & $\mathbf{R}^{\mathbf{2}}$ & $\mathbf{R}^{\mathbf{2}}$ Ajustado & Std Erro Estimado \\
\hline 1 & $0,433^{\text {a }}$ & 0,187 & 0,154 & 0,7173 \\
\hline
\end{tabular}

Fonte: Elaborado pelos autores (2019) 
As variáveis independentes respondem em 18,7\% a variável dependente, confirmando os estudos e pesquisas propostos por Walton (1973), Wang et al. (2010), Barbosa (2011), Sehrish e Zubair (2013), Covey (2014), De Paola e Gioia (2016), que relacionam a gestão do tempo com qualidade de vida. Destaca-se que os constructos apresentaram as seguintes médias e desvios padrões, conforme a Tabela 12.

Tabela 12: Média e desvio padrão dos constructos

\begin{tabular}{l|l|l}
\hline Constructos & Média & Desvio Padrão \\
\hline $\begin{array}{l}\text { Circunstância/ } \\
\text { Desperdício }\end{array}$ & 2.62 & 1.03 \\
\hline Importante & 3.08 & 1.12 \\
\hline Urgente & 2.70 & 1.07 \\
\hline
\end{tabular}

Fonte: Elaborado pelos autores (2019)

Diante dos dados da Tabela 12 é possível observar que o constructo Importância foi o que obteve maior média $(3,08)$ e também o maior desvio $(1,12)$, representando $36,66 \%$. Isso significa que os trabalhadores atribuem maior relevância, de fato, às atividades que são consideradas as mais importantes, como visitar parentes e amigos, tempo para se dedicar a si e às coisas que lhe interessam, como também à definição de objetivos e desenvolvimento de atividades físicas. As afirmações relacionadas à circunstância/desperdício e urgência obtiveram médias semelhantes, 2,62 e 2,70, assim como os desvios padrão, 1,03 e 1,07, respectivamente.

Em face da discussão articulada até então, observa-se que a QVT é influenciada diretamente pela GT, por meio dos constructos estudados, existindo várias formas de analisar o tempo, tais como, os modelos voltados para o desempenho (DAVIS, 2000; VAN EERDE, 2003; HÄFNER et al., 2015), para o bem-estar (MACAN et al., 1990; BRITTON; TESSER, 1991), e para o equilíbrio entre família e trabalho (WALTON, 1973; YEO; LI, 2012; SHAHBAZI et al., 2011; DE PAOLA; GIOIA, 2016). 


\section{CoNSIDERAÇão FINAIS}

Embora os aspectos envolvidos na QVT sejam muitos, a utilização de um construto que aborda a QVT a partir de questões relacionadas à GT acompanha uma tendência atual da gestão, uma vez que as demandas do ambiente laboral buscam amenizar os impactos da vida corrida, do estado de pressa, na produtividade e bem-estar, buscando o equilíbrio entre família e trabalho.

Com os resultados da pesquisa foi possível observar o quanto um bom planejamento por escrito é visto como essencial para a eficiente e sensata gestão do tempo. A aparente contradição entre entender o que é considerado importante, mas envolver-se em demandas emergentes e resolver questões pouco agregadoras de valor, justifica-se pelo aumento da pressão e redução dos prazos no trabalho, prejudicando a tomada de decisão.

Na análise da QVT com os construtos da GT, foi possível corroborar a ideia de que o tempo é melhor aproveitado com as coisas importantes quando não se está dedicado às circunstâncias/desperdícios e urgências. A maior relevância que os trabalhadores atribuem às atividades de seu real interesse e às pessoas de sua estima, bem como a importância que creditam à definição de objetivos e prática de atividades físicas, configuraram como aspectos fundamentais para a sua percepção de qualidade de vida.

Em tratando-se da contemporaneidade da temática GT tanto nas organizações, como no ambiente familiar, os resultados dessa pesquisa contribuem gerencialmente com as ações direcionadas para a QVT, de forma que os indivíduos estejam mais próximos de alcançar o equilíbrio entre os seus vários papeis sociais. Em relação às contribuições acadêmicas, a pesquisa contextualiza e integraliza outros estudos que abordam a relação QVT-GT, facilitando futuras investigações.

O estudo possui como limitação ter utilizado constructos que fazem parte da GT, mas que não foram validados conjuntamente em estudos anteriores, tratando do conteúdo sob os prismas da circunstância/desperdício, importância e urgência, e com tratamento estatístico.

A título de oportunidade de estudos futuros, indica-se a realização de pesquisas para comparar a QVT e GT a partir da percepção 
de gestores, tendo em vista o indicativo de maior pressão e tempo gasto no trabalho à medida que o nível hierárquico e as responsabilidades aumentam. Sugere-se também realizar comparações acerca da percepção da GT em outros ramos de atividade, bem como em outras regiões do país. Outra questão relevante é analisar a QVT e a GT no âmbito de pesquisas qualitativas, para entendimentos de informações subjetivas relacionadas as temáticas, para uma melhor tomada de decisão organizacional.

\section{REFERÊNCIAS}

ADAMS, Gary A.; JEX, Steve M. Relationships between time management, control, work-family conflict, and strain. Journal of occupational health psychology, v. 4, n. 1, p. 72, 1999.

AEON, Brad; AGUINIS, Herman. It's about time: New perspectives and insights on time management. Academy of management perspectives, v. 31, n. 4, p. 309-330, 2017.

AGUINIS, Herman; DAVIS, G. F.; DETERT, J. R.; GLYNN, M. A.; JACKSON, S. E.; KOCHAN, T.; KOSSEK, E. E.; LEANA C; Lee, T. W.; MORRINSON, E.; PEARCE, J.; PFEFFER, J.; ROUSSEAU, D.; SUTCLIFFE, K. M. Using organizational science research to address US federal agencies' management e labor needs. Behavioral Science \& Policy, v. 2, n. 2, p. 66-76, 2016.

ALAY, Sema; KOCAK, Settar. Validity and reliability of time management questionnaire. Hacettepe Üniversitesi Eğitim Fakültesi Dergisi, v. 22, n. 22, 2002.

ANCONA, Deborah G.; OKHUYSEN, G. A.; PERLOW, L. A. Time: A new research lens. Academy of management Review, v. 26, n. 4, p. 645-663, 2001.

BARBOSA, Christian. Triade Do Tempo, a. Elsevier Brasil, 2011.

BARNETT, Rosalind C.; BRENNAN, Robert T. Change in job conditions, change in psychological distress, and gender: A longitudinal study of dual-earner couples. Journal of Organizational Behavior: The International Journal of Industrial, Occupational and Organizational Psychology and Behavior, v. 18, n. 3, p. 253-274, 1997.

BIRD, B. J.; JORDAN, R. S. A study to develop measures of time orientation and future time perspective. In: meeting of the Academy of Management, New Orleans, LA. 1987.

BLUEDORN, Allen C.; KAUFMAN, Carol Felker; LANE, Paul M. How many things do you like to do at once? An introduction to monochronic and polychronic time. Academy of Management Perspectives, v. 6, n. 4, p. 17-26, 1992.

BOND, Michael J.; FEATHER, N. T. Some correlates of structure and purpose in the use of time. Journal of personality and social psychology, v. 55, n. 2, p. 321, 1988.

BRITTON, Bruce K.; TESSER, Abraham. Effects of time-management practices on college grades. Journal of educational psychology, v. 83, n. 3, p. 405, 1991. 
BROWN, Timothy A. Confirmatory factor analysis for applied research. Guilford publications, 2014.

BURRUS, Jeremy; JACKSON, T., HOLTZMAN, S.; ROBERTS, R. D. Teaching high school students to manage time: The development of an intervention. Improving Schools, v. 20, n. 2, p. 101-112, 2017.

BURT, Christopher DB; KEMP, Simon. Construction of activity duration and time management potential. Applied Cognitive Psychology, v. 8, n. 2, p. 155-168, 1994.

CLAESSENS, Brigitte JC; VAN EERDE, W.; RUTTE, C. G.; ROE, R. A. Planning behavior and perceived control of time at work. Journal of Organizational Behavior: The International Journal of Industrial, Occupational and Organizational Psychology and Behavior, v. 25, n. 8, p. 937-950, 2004.

276, 2007.

. A review of the time management literature. Personnel Review, v. 36, n. 2, p. 255-

COOPER, Donald R.; SCHINDLER, Pamela S. Métodos de Pesquisa em Administração-12 edição. McGraw Hill Brasil, 2016.

COVEY, Stephen R. The 7 habits of highly effective families. St. Martin's Press, 2014.

CRESWELL, John W.; CRESWELL, J. David. Research design: Qualitative, quantitative, and mixed methods approaches. Sage publications, 2017.

DAL FORNO, C.; FINGER, I. R. Qualidade de vida no trabalho: conceito, histórico e relevância para a gestão de pessoas. Programa de Pós-Graduação em Engenharia de Produção-PPGEP Laboratório de Qualidade de Vida-LaQVida Universidade Tecnológica Federal do ParanáUTFPR Ponta Grossa-PR-Brasil, v. 7, n. 02, p. 103-112, 2015.

DAVIS, M. A. Time and the nursing home assistant: Relations among time management, perceived control over time, and work-related outcomes. Academy of Management, Toronto, Canada, 2000.

DE PAOLA, Maria; GIOIA, Francesca. Who performs better under time pressure? Results from a field experiment. Journal of Economic Psychology, v. 53, p. 37-53, 2016.

DEVELLIS, Robert F. Scale development: Theory and applications. Sage publications, 2016. DRUCKER, Peter F. The Efective Executive. 1967.

EISINGA, Rob; TE GROTENHUIS, Manfred; PELZER, Ben. The reliability of a two-item scale: Pearson, Cronbach, or Spearman-Brown? International journal of public health, v. 58, n. 4, p. 637-642, 2013.

FIELD, Andy P.; MILES, Jeremy; FIELD, Zoë. Discovering statistics using R/Andy Field, Jeremy Miles, Zoë Field. 2012.

FRANCIS-SMYTHE, Jan A.; ROBERTSON, Ivan T. On the relationship between time management and time estimation. British Journal of Psychology, v. 90, n. 3, p. 333-347, 1999.

GARHAMMER, Manfred. Pace of life and enjoyment of life. Journal of Happiness Studies, v. 3, n. 3, p. 217-256, 2002. 
GEORGE, Jennifer M.; JONES, Gareth R. The role of time in theory and theory building. Journal of management, v. 26, n. 4, p. 657-684, 2000.

GRANT, Adam M.; CHRISTIANSON, Marlys K.; PRICE, Richard H. Happiness, health, or relationships? Managerial practices and employee well-being tradeoffs. Academy of management perspectives, v. 21, n. 3, p. 51-63, 2007.

GREENHAUS, Jeffrey H.; COLLINS, Karen M.; SHAW, Jason D. The relation between workfamily balance and quality of life. Journal of vocational behavior, v. 63, n. 3, p. 510-531, 2003.

GUEST, Robert H. Quality of work life, learning from Tarrytown. Harvard University Graduate School of Business Administration, 1979.

HAIR, J. F.; BLACK, W. C.; BABIN, B. J.; ANDERSON, R. E.; Tatham, R. L. Multivariate data analysis (Vol. 6). 2006.

HÄFNER, Alexander; STOCK, A., PINNEKER, L., STROHLE, S. Stress prevention through a time management training intervention: An experimental study. Educational Psychology, v. 34, n. 3, p. 403-416, 2014.

HÄFNER, Alexander; STOCK, Armin; OBERST, Verena. Decreasing students' stress through time management training: An intervention study. European journal of psychology of education, v. 30, n. 1, p. 81-94, 2015.

JACKSON, Paul R.; MARTIN, Robin. Impact of just-in-time on job content, employee attitudes and well-being: a longitudinal study. Ergonomics, v. 39, n. 1, p. 1-16, 1996.

KÄSER, Philipp AW; FISCHBACHER, Urs; KÖNIG, Cornelius J. Helping and quiet hours: Interruption-free time spans can harm performance. Applied Psychology, v. 62, n. 2, p. 286-307, 2013.

KAUFMAN-SCARBOROUGH, Carol; JAY, D. Lindquist. Time management and polychronicity. Comparisons, contrasts, and insights for the workplace. Journal of Managerial Psychology, v. 14, n. 3-4, p. 288-312, 1999.

$\mathrm{KOCH}$, Cornelius J.; KLEINMANN, Martin. A stitch in time saves nine: Behavioural decision-making explanations for time management problems. European Journal of Work and Organizational Psychology, v. 11, n. 2, p. 199-217, 2002.

LAKEIN, Alan; LEAKE, Pip. How to get control of your time and your life. New York: New American Library, 1973.

LANDY, Frank J.; RASTEGARY, H.; THAYER, J.; Colvin, C. Time urgency: The construct and its measurement. Journal of Applied Psychology, v. 76, n. 5, p. 644, 1991.

LEE, Heejin; LIEBENAU, Jonathan. A New Time Discipline: Managing. Making time: Time and management in modern organizations, p. 126, 2002.

LIMONGI FRANÇA, Ana Cristina. Qualidade de vida no trabalho: conceitos e práticas nas empresas da sociedade pós-industrial. In: Qualidade de vida no trabalho: conceitos e práticas nas empresas da sociedade pós-industrial. 2004.

MACAN, Therese H.; SHAHANI, C.; DIPBOYE, R. L.; PHILLIPS, A. P. College students' time management: Correlations with academic performance and stress. Journal of educational psychology, v. 82, n. 4, p. 760, 1990. 
MACAN, Therese Hoff. Time management: Test of a process model. Journal of applied psychology, v. 79, n. 3, p. 381, 1994.

MACAN, Therese Hoff. Time-management training: Effects on time behaviors, attitudes, and job performance. The Journal of psychology, v. 130, n. 3, p. 229-236, 1996.

MACKENZIE, Alec; NICKERSON, Pat. The time trap: The classic book on time management. Amacom, 2009.

MADJAR, Nora; OLDHAM, Greg R. Task rotation and polychronicity: Effects on individuals' creativity. Human Performance, v. 19, n. 2, p. 117-131, 2006.

MAJOR, Virginia Smith; KLEIN, Katherine J.; EHRHART, Mark G. Work time, work interference with family, and psychological distress. Journal of applied psychology, v. 87, n. 3, p. $427,2002$.

MALHOTRA, Naresh K. Pesquisa de Marketing-: Uma Orientação Aplicada. Bookman Editora, 2001.

MCCAY, J. The Management of Time. New Jersey: Prentice Hall, Englewood Cliffs, 1959.

MYSZEWSKI, Jan M. No time for quality. TQM Journal, v. 27, n. 4, p. 435, 2015.

NADLER, David A.; LAWLER, Edward E. Quality of work life: Perspectives and directions. Organizational dynamics, 1983.

ORLIKOWSKI, Wanda J.; YATES, JoAnne. It's about time: Temporal structuring in organizations. Organization science, v. 13, n. 6, p. 684-700, 2002.

PALMER, David K.; SCHOORMAN, F. David. Unpackaging the multiple aspects of time in polychronicity. Journal of Managerial Psychology, 1999.

PARKES, Katharine R.; MENDHAM, Clare A.; VON RABENAU, Caroline. Social support and the demand-discretion model of job stress: Tests of additive and interactive effects in two samples. Journal of Vocational Behavior, v. 44, n. 1, p. 91-113, 1994.

PEETERS, Miranda A. G; RUTTE, Christel G. Time management behavior as a moderator for the job demand-control interaction. Journal of occupational health psychology, v. 10, n. 1, p. 64, 2005.

POPOSKI, Elizabeth M.; OSWALD, Frederick L.; BROU, Randy J. Development of a new measure of polychronicity. Michigan State University East Lansing, 2009.

RICHARDS, J. H. Time management-a review. Work \& Stress, v. 1, n. 1, p. 73-78, 1987.

RICHARDSON, Roberto Jarry. Pós-Graduação-Metodologia-Pesquisa Social: Métodos e Técnicas-Métodos Quantitativos e Qualitativos-Capitulo 5. Editora ATLAS SA-2015-São Paulo, 2017.

ROBERTS, R. D.; KRAUSE, H.; SUK-LEE, L. Australian time organization and management scales. Unpublished Inventory: University of Sydney, 2001.

SEHRISH, Javeria; ZUBAIR, Aisha. Polychronicity, time management and work related quality of life among bank employees. Pakistan Journal of Psychology, v. 44, n. 2, 2013. 
SHAHBAZI, Behzad; SHOKRZADEH S.; BEJANI, H.; MALEKINIA, E.; GHORONEH, D. A survey of relationship between the quality of work life and performance of Department Chairpersons of Esfahan University and Esfahan Medical Science University. Procedia-Social and Behavioral Sciences, v. 30, p. 1555-1560, 2011.

SLOCOMBE, Thomas E.; BLUEDORN, Allen C. Organizational behavior implications of the congruence between preferred polychronicity and experienced work-unit polychronicity. Journal of Organizational Behavior: The International Journal of Industrial, Occupational and Organizational Psychology and Behavior, v. 20, n. 1, p. 75-99, 1999.

TEUCHMANN, Katja; TOTTERDELL, Peter; PARKER, Sharon K. Rushed, unhappy, and drained: an experience sampling study of relations between time pressure, perceived control, mood, and emotional exhaustion in a group of accountants. Journal of occupational health psychology, v. 4, n. 1, p. 37, 1999.

VAN EERDE, Wendelien. Procrastination at work and time management training. The Journal of psychology, v. 137, n. 5, p. 421-434, 2003.

WALTON, Richard E. Quality of working life: what is it. Sloan management review, v. 15, n. 1, p. 11-21, 1973.

WANG, Wei-Ching; KAO, C. H.; HUAN, T. C.; WU. Free time management contributes to better quality of life: A study of undergraduate students in Taiwan. Journal of Happiness Studies, v. 12, n. 4, p. 561-573, 2011.

WHITE, Suzanne M.; RILEY, Anne; FLOM, Peter. Assessment of Time Management Skills (ATMS): A practice-based outcome questionnaire. Occupational Therapy in Mental Health, v. 29, n. 3, p. 215-231, 2013.

YEO, Roland K.; LI, Jessica. In pursuit of learning: sensemaking the quality of work life. European Journal of Training and Development, 2013.

Recebido em: 30-1-2020

Aprovado em: 12-12-2020

Avaliado pelo sistema double blind review.

Disponível em http://mjs.metodista.br/index.php/roc 